\title{
High Serum FSH is Associated with Brown Oocyte Formation and a Lower Pregnacy Rate in Human IVF Parctice
}

\author{
Hongyi Xu Kai Denga,b Qingbing Luo ${ }^{a}$ Juan Chen ${ }^{a} \quad$ Xin Zhang ${ }^{a} \quad$ Xiaoyan Wang ${ }^{a, b}$ \\ Honglu Diao ${ }^{a}$ Changjun Zhang ${ }^{a}$
}

aReproductive Medicine Center, Renmin Hospital, Hubei University of Medicine, Shiyan, China;

bWellcome Trust Centre for Human Genetics, University of Oxford, Oxford, United Kingdom

\section{Key Words}

Brown oocyte $•$ Zona pellucida $\cdot$ IVF-ET $•$ FSH level $•$ Fertilization outcome

\begin{abstract}
Background/Aims: To investigate whether brown zona pellucida (ZP) of oocytes affects the outcome of fertilization, embryo quality and pregnancy rate in in vitro fertilization-embryo transfer (IVF-ET). Methods: Based on the ZP color of their oocytes, a total number of 703 patients dated from 2012 to 2014 were divided into a normal egg group (group A) and a brown oocyte group (group B), with 629 and 74 cases, respectively. Clinical characteristics, gonadotropin $(\mathrm{Gn})$ days, Gn dosage, serum hormone levels on the day of human chorionic gonadotropin (HCG) injection, ZP thickness (ZPT) of the eggs, fertilization rate, rescue intracytoplasmic sperm injection (rICSI) rate, good-quality embryo rate and pregnancy rate were compared between the two groups. Results: No significant differences were found in the duration and the causes of infertility, and their basal level of endocrine hormone before IVFET between normal egg group and brown egg group. The level of serum hormone including estradiol, progesterone and luteinizing hormone on the day of HCG injection were again similar. Moreover, there were no differences in number of mature oocytes, oocyte fertilization rates and rICSI rates after IVF between the two groups. However, we observed that the ZPT of brown oocytes (group B) was higher than that of normal oocytes (group A). Moreover, the Gn dosage and FSH levels on the day of HCG injection were significantly higher in group $B$ than in group $A$ and the good-quality embryo rate and pregnancy rate in group $B$ were lower than those in group A. Conclusion: Compared with normal eggs, oocytes with a brown ZP were found to have a higher ZPT, lower embryo quality and lower pregnancy rate, which might be due to a high $\mathrm{Gn}$ dosage injection and high serum FSH levels during IVT-ET cycles.
\end{abstract}




\section{Introduction}

Acquisition of high-quality embryos is very important for improving the clinical pregnancy rate in in vitro fertilization-embryo transfer (IVF-ET). The oocyte quality is one of the key factors affecting implantation and pregnancy rates in human assisted reproductive technology (ART). Currently, the morphological evaluation is the most objective method to assess the quality of oocyte, and morphological features of eggs are also being used to predict the potential of embryonic development [1]. Good quality MII oocytes are judged based on several features such as a clear perivitelline space with certain thickness, well-distributed cytoplasmic granular particles, as well as a complete first polar body and a pale-colored zona pellucida (ZP) [2]. Depending on the locations of abnormal morphologies, Oocyte morphological abnormalities can be divided into intra-cytoplasmic and extra-cytoplasmic abnormalities. Cytoplasmic abnormalities include an abnormal distribution of cytoplasmic granules, abnormalities of the smooth endoplasmic reticulum and a liquefied bubble. Extracytoplasmic abnormalities include an irregular oocyte shape, enlargement of the perivitelline space, fragments in the perivitelline space and abnormalities in oocyte membrane viscosity and the ZP color, shape or thickness.

No consensus has been reached about the effects of oocyte morphological anomalies on fertilization, embryo quality and pregnancy outcomes [3]. Some studies have shown that abnormal oocytes adversely affected fertilization, embryo quality and pregnancy outcomes [4-7]: the pregnancy and implantation rates decline with the transfer of embryos developed from morphologically abnormal oocytes $[8,9]$. In contrast, other studies have shown that oocytes with morphological abnormalities did not affect the fertilization outcome [1012] and the developmental potential of an embryo cannot be predicted from its oocyte morphology unless the morphology is extremely abnormal [13].

Maturation of the human follicle can be divided into four stages: the primordial follicle, the primary follicle, secondary follicle and mature follicle, regardless of in a natural cycle or stimulated ovulation cycle. Any anomalies during these steps can block development of follicular and cause morphological abnormalities in the oocyte. During the process of oocyte maturation, the ZP changes constantly. Under most circumstances, the ZP appears to be pale, while in some cases the ZP appears to be brown or black. Oocytes with this brown or black colored ZP are called brown oocyte. Whether the color of ZP affect the outcome of pregnancy remains unknown. Some reports showed that brown oocytes had no significant effect on pregnancy outcomes [14], while others have suggested that a brown oocyte could be associated with a lower fertilization, implantation or pregnancy rate [15].

The exact reasons for the ZP color change remain unknown. In this study, we collected data for all brown oocytes in the past two years (2012 - 2014) from our IVF center in Hubei Province, China, and compared their clinical features, the use of chelating agents, and basal hormone and daily HCG hormone levels. We analyzed the potential reasons leading to the ZP color change and its influence on the fertilization rate, embryo quality and pregnancy rate.

\section{Materials and Methods}

\section{Study population}

A retrospective analysis of 703 patients who underwent IVF-ET cycles in the Reproductive Medicine Center, Renmin Hospital, Hubei University of Medicine from February 2012 to December 2014 was performed. These patients were aged 22-40 years and had infertility for 2-8 years, excluding male infertility. Of the 703 patients, 468 cases were due to tubal infertility, 153 ovulation failure, 32 endometriosis, 23 polycystic ovary syndrome (PCOS), 2 immune infertility, and 25 unexplained infertility. The patients were divided into two groups based on the ZP color of their oocytes, with 629 cases with normal colored oocytes in Group A (Fig. 2, right panel), and 74 cases with brown oocytes in Group B (Fig. 2, left panel). The oocytes appeared to be brown due to the brown color of the ZP, as shown in Fig. 3, which shows a zygote that had a ZP separated from the oocyte cytoplasm. The study was approved by the Human Reproductive Medical Ethics Committee of Shiyan Renmin Hospital with written informed consent obtained from all subjects. 


\section{Cellular Physiology Cell Physiol Biochem 2016;39:677-684 and Biochemistry Published online: July 21, $2016 \quad \begin{aligned} & \text { DOI: 10.1159/000445658 } 2016 \text { The Author(s). Published by S. Karger AG, Basel } \\ & \text { www.karger.com/cpb }\end{aligned}$ \\ Xu et al.: High FSH Caused Brown Oocyte and a Lower Pregnacy Rate}

Ovarian stimulation and follicle growth monitoring

The gonadotropin-releasing hormone (GnRH) long protocol was used for all patients [16], with daily injections of $0.05 \mathrm{mg} /$ day triptorelin beginning in the luteal phase of the last menstrual cycle, to downregulate the activity of pituitary gland. Gonal-F alone or with human menopausal gonadotropin (HMG) was used for ovarian stimulation. The follicle was monitored using ultrasound until it reached 16-18 mm in diameter and was then stopped using FSH and HMG. An intramuscular injection of 5000-10000 IU of human chorionic gonadotropin (HCG) was given in 36 hrs before retrieval of the follicles along with the oocyte through transvaginal ultrasonography.

\section{Measurement of hormones concentration}

Venous blood was drawn in the morning of the second day of the menstrual cycle of the controlled ovarian hyperstimulation ( $\mathrm{COH}$ ) cycle of the day of HCG administration. FSH, E2, P and LH were measured by the DXI800 automatic electrochemical luminescence immunoassay system. The Access ESTRADIOL 3340 kits were purchased from Beckman Coulter, Inc. (Chaska, USA).

Oocyte observation and fertilization assessment

All of the oocytes were fertilized by IVF and the quality of their oocytes were assessed by two professional embryologists. A capillary was used to mechanically strip granule cells around the oocyte. The color of oocytes were observed. Whether the second polar body had discharged after 4 6 hours of fertilization is also been observed. Oocytes with a brown ZP were considered as brown oocytes (Fig. 2, left panel). Fertilization was only considered to be successful when all of the oocytes were observed to have extruded their second polar body. Otherwise, a rescue intracytoplasmic sperm injection (rICSI) procedure was subsequently performed and the fertilization outcome was judged base on the appearance of pronucleus after 16-18 hrs. If the two pronuclear stage appeared, we re-considered the fertilization to be successful.

\section{Embryo transfer technique}

The procedure of embryo transfer was performed as following. Firstly, a $1 \mathrm{ml}$ syringe was connected to the embryo transfer catheter. Then, we transferred the embryos in turn, as follows: $5 \mu$ l culture media, $5 \mu \mathrm{l}$ air, 8-10 $\mu \mathrm{l}$ culture media containing the embryos, $5 \mu \mathrm{l}$ air, $5 \mu \mathrm{l}$ culture media. Finally, we delivered the embryos into the uterus. In this procedure, the embryologist must check carefully and ensure that no embryo is left in the tube under the microscope.

\section{Embryo culture and pregnancy assessment}

Following fertilization by IVF or ICSI, the zygotes were transferred to the embryo culture media for 24 $48 \mathrm{hrs}$. The embryo morphological grading was performed on day 3 after ovum pick-up (OPU) [17]. Embryos with a magnitude of III or above were viable. Embryos with good morphology such as homogeneously distributed blastomeres and less fragments were usually preferred for transfer. We transplanted 2-3 embryos using ultrasound-guided embryo intrauterine transfer based on patient's age. After fourteen days of the embryo transplantation, the biochemical pregnancy could be detected and the clinical pregnancy is determined by successful detection of fetal heartbeat by ultrasound four weeks after embryo transfer.

\section{Statistical Analysis}

The data on concentrations of hormones and pregnancy rates were processed using SPSS 13.0 statistical software. Measurement data are described as the means \pm sd, and differences between the groups were compared using the two-sided t test. The rates were compared using a Chi square test, where appropriate. Differences with P-values of $<0.05$ were considered to be statistically significant.

\section{Results}

General comparison of patients in the brown oocyte group and the normal group

6742 oocytes, of which 5824 were mature, were collected from 703 patients and divided into two groups based on the color of oocytes. No significant differences were observed for the average age, body mass index (BMI), duration or causes of infertility between patients in the brown oocyte and normal groups (Table 1). 
Table 1. Comparison of clinical features between the normal (group A) and brown oocyte (group B) groups (\% $\overline{\mathrm{x}} \pm \mathrm{S}$ ). * BMI, body mass index

\begin{tabular}{lllll}
\hline Factors & & Group A (n=629) & Group B (n=74) & P-value \\
\hline Maternal age (years) & Average age & $30.6 \pm 4.0$ & $31.54 \pm 3.2$ & 0.381 \\
& $22-25$ & $66(10.49 \%)$ & $6(8.11 \%)$ & 0.685 \\
& $26-30$ & $292(46.42 \%)$ & $30(40.54 \%)$ & 0.388 \\
& $31-35$ & $208(33.07 \%)$ & $30(40.54 \%)$ & 0.125 \\
& $36-40$ & $63(10.02 \%)$ & $8(10.81 \%)$ & 0.818 \\
BMI* & & $22.4 \pm 2.9$ & $22.3 \pm 3.0$ & 0.615 \\
Duration of infertility (years) & $4.1 \pm 3.0$ & $3.8 \pm 2.6$ & 0.213 \\
Primary infertility & & $268 / 629(42.6 \%)$ & $32 / 74(43.2 \%)$ & 0.514 \\
Secondary infertility & & $361 / 629(57.4 \%)$ & $42 / 74(56.8 \%)$ & 0.522 \\
Tubal factor & $423 / 629(67.2 \%)$ & $45 / 74(60.8 \%)$ & 0.438 \\
Ovulatory disorder & $135 / 629(21.5 \%)$ & $18 / 74(24.3 \%)$ & 0.372 \\
Endometriosis & $29 / 629(4.6 \%)$ & $3 / 74(4.1 \%)$ & 0.563 \\
Immune infertility & $2 / 629(0.3 \%)$ & $0 / 74(0 \%)$ & 0.801 \\
Unexplained & $21 / 629(3.3 \%)$ & $4 / 74(5.4 \%)$ & 0.275 \\
\hline
\end{tabular}

Table 2. Comparison of the stimulated ovulation process between the two groups ( $\% \overline{\mathrm{x}} \pm \mathrm{S})$. * Number of Gn, $75 \mathrm{U} / \mathrm{ampule.} \mathrm{FSH,} \mathrm{Fol-}$ licle-stimulating hormone; LH, Luteinizing hormone; E2, Estradiol; P, progesterone

\begin{tabular}{|c|c|c|c|}
\hline Factors & Group A (n=629) & Group B $(n=74)$ & P-value \\
\hline \multicolumn{4}{|c|}{ Basal endocrine hormone levels } \\
\hline FSH (IU/L) & $9.0 \pm 3.9$ & $9.8 \pm 5.4$ & 0.131 \\
\hline LH (IU/L) & $4.9 \pm 1.8$ & $5.0 \pm 2.0$ & 0.546 \\
\hline $\mathrm{E}_{2}(\mathrm{nmol} / \mathrm{L})$ & $55.0 \pm 10.2$ & $57.7 \pm 11.2$ & 0.158 \\
\hline$P(\rho m o l / L)$ & $3.7 \pm 0.6$ & $4.0 \pm 0.4$ & 50.236 \\
\hline Days of Gn & $10.4 \pm 1.6$ & $12.5 \pm 2.4$ & 0.212 \\
\hline Number of Gn* & $24.2 \pm 11.6$ & $32.5 \pm 10.6$ & 0.019 \\
\hline \multicolumn{4}{|c|}{ Day of HCG injection } \\
\hline FSH (IU/L) & $13.6 \pm 6.1$ & $17.5 \pm 8.1$ & 0.02 \\
\hline LH (IU/L) & $1.9 \pm 1.0$ & $1.2 \pm 0.5$ & 0.246 \\
\hline $\mathrm{E}_{2}(\mathrm{nmol} / \mathrm{L})$ & $4251.2 \pm 604.5$ & $4058.1 \pm 825.5$ & 0.703 \\
\hline$P(\rho \mathrm{mol} / \mathrm{L})$ & $4.5 \pm 0.5$ & $4.7 \pm 0.5$ & 0.072 \\
\hline
\end{tabular}

Comparison of drug dosage and serum hormones in superovulation between two groups

As shown in Table 2, no significant $(\mathrm{P}>0.05)$ differences were found between the two groups in basal endocrine hormone levels on day 3 ; in the average number of days with medicine; or in E2, LH, or P levels on the day of HCG injection. However, the differences in the average Gn count and the serum FSH levels on the day of HCG administration were significant: $13.6 \pm 6.1$ in normal group versus $17.5 \pm 8.1$ in brown oocytes group $(\mathrm{P}<0.05)$.

Comparison of fertilization outcomes between the two groups

2819 high-quality embryos were successfully generated from 5824 mature oocytes. There were no significant $(P>0.05)$ differences in the average number of eggs, mature egg rate, fertilization rate, rICSI rate and good-quality embryo rate between the brown oocytes 


\section{Cellular Physiology Cell Physiol Biochem 2016;39:677-684 \begin{tabular}{l|l} 
and Biochemistry Published online: July 21, 2016 & $\begin{array}{l}\text { D } 2016 \text { The Author(s). Published by S. Karger AG, Basel } \\
\text { www.karger.com/cpb }\end{array}$
\end{tabular} \\ Xu et al.: High FSH Caused Brown Oocyte and a Lower Pregnacy Rate}

Table 3. Comparison of fertilization outcomes between the two groups ( $\% \overline{\mathrm{x}} \pm \mathrm{S}$ ). * The oocyte maturation process in MII stage; ${ }^{* *} 2$-pronuclear stage; ***Rescue ICSI, Rescue intracytoplasmic sperm injection

\begin{tabular}{llll}
\hline Factors & Group A (n=629) & Group B (n=74) & P-value \\
\hline Number of oocytes & $9.5 \pm 9.2$ & $10.0 \pm 8.1$ & 0.606 \\
Number of embryos transferred & $2.2 \pm 0.5$ & $2.2 \pm 0.5$ & 0.684 \\
Zona pellucida thickness $(\mu \mathrm{m})$ & $17.3 \pm 6.3$ & $20.9 \pm 7.0$ & 0.572 \\
Rate of MII* & $86.4 \%(5160 / 5970)$ & $85.9 \%(664 / 773)$ & 0.521 \\
Rate of 2PN** & $78.7 \%(4701 / 5970)$ & $79.6 \%(615 / 773)$ & 0.852 \\
Abnormal fertilization rate & $6.0 \%(359 / 5970)$ & $5.4 \%(42 / 773)$ & 0.545 \\
Rescue ICSI rate*** & $4.1 \%(26 / 629)$ & $6.8 \%(5 / 74)$ & 0.325 \\
Cleavage rate & $99.5 \%(4677 / 4701)$ & $99.2 \%(610 / 615)$ & 0.989 \\
Rate of embryos available for transfer & $67.1 \%(3138 / 4677)$ & $61.3 \%(374 / 610)$ & 0.120 \\
Rate of good-quality embryos & $54.7 \%(2560 / 4677)$ & $42.5 \%(259 / 610)$ & 0.007 \\
Clinical pregnancy rate & $63.4 \%(399 / 629)$ & $39.2 \%(29 / 74)$ & 0.030 \\
\hline
\end{tabular}

group and the normal group (Table 3). However, the average ZP thickness of the brown oocyte group was higher than that of the normal group $(\mathrm{P}<0.05)$, and the high-quality embryo and clinical pregnancy rates were lower than those of the normal group $(\mathrm{P}<0.05)$.

\section{Discussion}

Compared with normal oocytes, the ZP of the brown oocytes showed abnormal color changes and were a translucent brown color in our study. There are few reports on the causes of or the mechanisms for the formation of brown oocytes. Our study suggests that the possible cause of the formation of oocytes with a brown ZP is not related to the average age of the infertile patients or to the cause and duration of infertility. Previous studies have shown no significant correlation between color of oocytes and the clinical outcome of pregnancy but have suggested that brown oocytes could be caused by ovarian stimulation [18].

Exogenous FSH is commonly used to accelerate the maturation of follicles in order to obtain sufficient amount of oocytes in IVF cycles, in which the high-quality embryos can be selected after IVT for transfer. Exogenous FSH may affect the differentiation and growth of granulosa cells as well as the development of ovarian follicles. The appropriate FSH dosage does not affect gene expression of oocytes and granular cells significantly. However, a high FSH dosage will alter pattern of gene expression in antral follicular and granular cells, which usually results in disfunction of granular cell and abnormal oocyte development. Studies have shown that a high dose of exogenous hormones, especially FSH administrated in IVFET cycles, induce the morphologically abnormality of oocytes, including abnormalities in the ZP, cytoplasm and polar body [19]. Min Li et al. showed that the effect of exogenous FSH on oocytes and embryos is dependent on its concentration and that high-dose FSH reduced the quality of oocytes and embryos [20].

In this study, most granulosa cells of brown oocytes were well scattered but were dark and aggregated (Fig. 1), indicating that the granulosa cells may have been premature. ZP is mainly composed of glycoproteins (ZP1, ZP2 and ZP3 in mice), which are secreted by oocytes and granulosa cells. By knocking out ZP glycoprotein gene ZP1 in mice embryonic stem cells, Rankin found that the ZP structure of their oocytes was looser with enlarged perivitelline space [21]. In addition, targeted mutagenesis of ZP1 also resulted in heterogeneous distribution of granulosa cells around the oocyte, and incomplete ZP matrix. These changes suggested abnormal synthesis or secretion of the ZP protein from granulosa cells [22]. In our results, the occurrence of brown ZP correlate with a high dosage of exogenous FSH, 


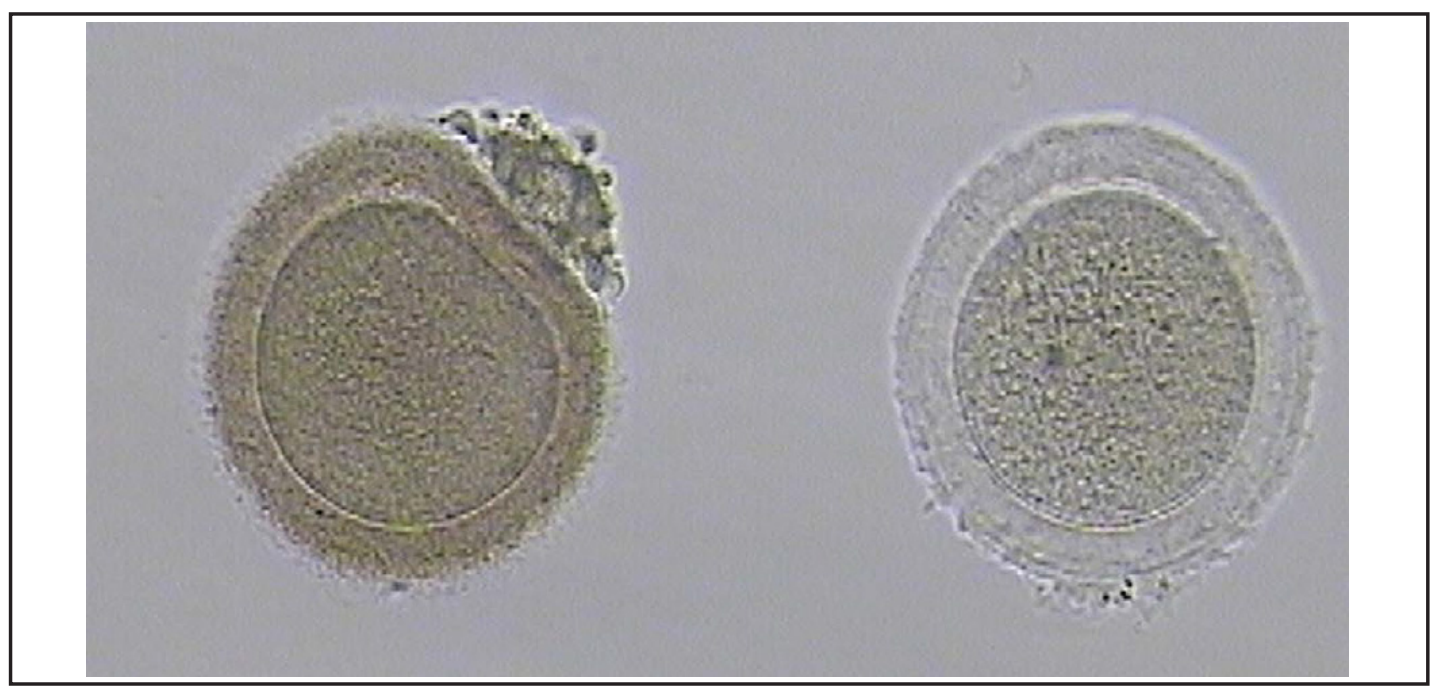

Fig. 1. Cumulus oophorus of oocyte with a brown zona pellucida $(4 \times)$.

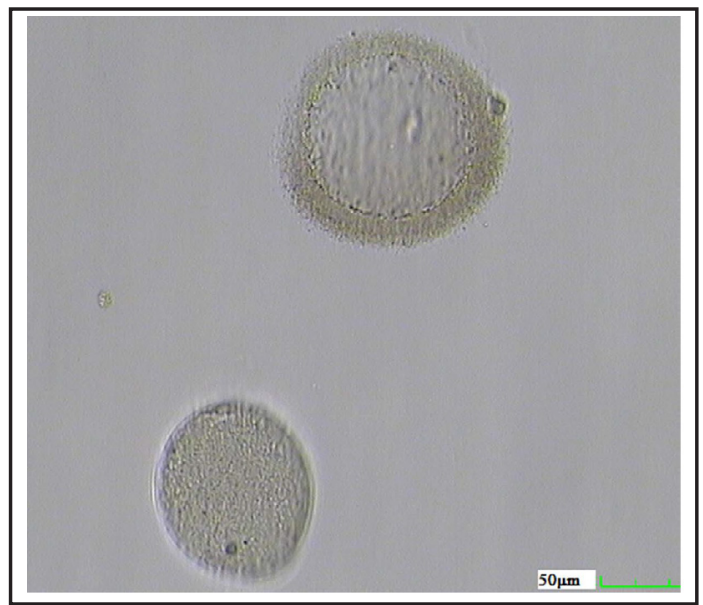

Fig. 2. Brown oocyte (left) and normal oocyte (right) (20x).

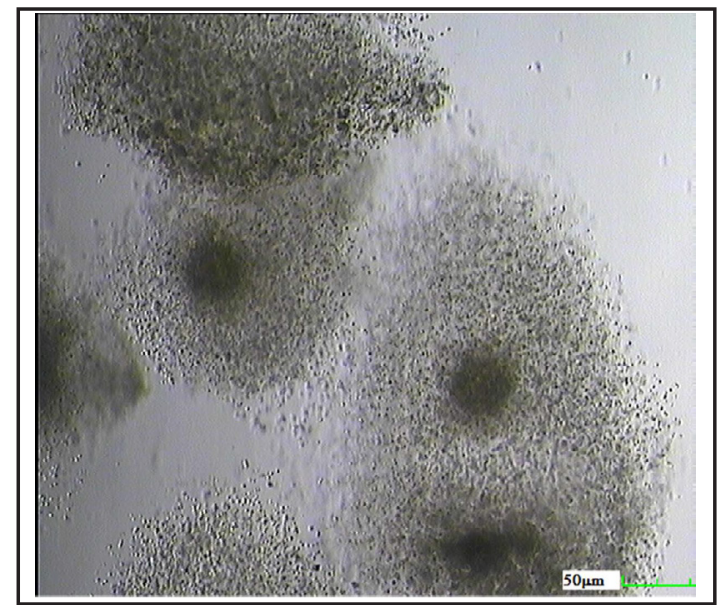

Fig. 3. Fertilized ovum with a brown zona pellucida $(20 \times)$.

indicating that the high dosage of exogenous FSH might lead to the brown ZP. It is possible that the high dosage of exogenous FSH continuously enters into the follicular fluid, leading to the abnormal synthesis and secretion of ZP protein from granulosa cells. Ultimately, this leads to the premature granular cells, with excessively dispersed or agglutinated appearance.

As shown in Table 3, the number of high-quality embryos and pregnancy rate of the brown oocyte group were significantly lower than those of the normal oocyte group, which indicated that brown oocytes affected embryo development. In our study, clinical pregnancy was defined as the presence of an intrauterine gestational sac on the trans-vaginal ultrasound examination. We did not measure AMH concentrations [23-25].

Most of the brown oocytes have a thicker ZP which appear darker than normal ones. ZP thickness in individual preembryos has been proposed to be a marker of normal human preembryo development because a correlation was observed between ZP thickness and implantation of thawed as well as fresh preembryos [26, 27]. A thinner portion of the zona pellucida is very likely necessary for appropriate hatching during the blastocyst stage. Thicker ZP preembryos reduced the implantation potential during hatching, and as a result, the high-quality embryo rate and clinical pregnancy rate are lower. We have incomplete data regarding live birth.

\section{KARGER}




\section{Cellular Physiology Cell Physiol Biochem 2016;39:677-684 \begin{tabular}{ll|l} 
DOI: 10.1159/000445658 & $\begin{array}{l}\text { O 2016 The Author(s). Published by S. Karger AG, Basel } \\
\text { www.karger.com/cpb }\end{array}$
\end{tabular} \\ Xu et al.: High FSH Caused Brown Oocyte and a Lower Pregnacy Rate}

In conclusion, high serum FSH levels during the IVF-ET cycle were correlated with the formation of brown oocytes and affected embryo development, leading to a decrease in the pregnancy rate. In contrast, no significant correlation was observed between brown oocytes formation and patients' conditions like age, BMI, or duration and causes of infertility. Therefore, limiting the FSH dose during controlled ovarian hyperstimulation is important for the production of high-quality oocytes.

\section{Acknowledgments}

This research was financially supported by grants from the National Natural Science Foundation of China (81401200), the Natural Science Foundation of Hubei Province of China (2013CFB479), Shiyan science and technology research and development projects (15Y41/14K69) and the Key Discipline Project of Hubei Province (2014XKJSSJ08).

\section{Disclosure Statement}

All authors are in agreement with the content of this manuscript. The authors declare no conflict of interest.

\section{References}

1 Wilding M, Di Matteo L, D'Andretti S, Montanaro N, Capobianco C, Dale B: An oocyte score for use in assisted reproduction. J Assist Reprod Genet 2007;24:350-358.

2 Veeck LL: Oocyte assessment and biological performance. Ann N Y Acad Sci 1988;541:259-274.

3 Rienzi L, Vajta G, Ubaldi F: Predictive value of oocyte morphology in human IVF: a systematic review of the literature. Hum Reprod Update 2011;17:34-45.

4 Alikani M, Palermo G, Adler A, Bertoli M, Blake M, Cohen J: Intracytoplasmic sperm injection in dysmorphic human oocytes. Zygote 1995;3:283-288.

5 Ten J, Mendiola J, Vioque J, de Juan J, Bernabeu R: Donor oocyte dysmorphisms and their influence on fertilization and embryo quality. Reprod Biomed Online 2007;14:40-48.

6 Rienzi L, Balaban B, Ebner T, Mandelbaum J: The oocyte. Hum Reprod 2012;27:i2-21.

7 Van Blerkom J, Henry G: Oocyte dysmorphism and aneuploidy in meiotically mature human oocytes after ovarian stimulation. Hum Reprod 1992;7:379-390.

8 Xia P: Intracytoplasmic sperm injection: correlation of oocyte grade based on polar body, perivitelline space and cytoplasmic inclusions with fertilization rate and embryo quality. Hum Reprod 1997;12:17501755.

9 Meriano JS, Alexis J, Visram-Zaver S, Cruz M, Casper RF: Tracking of oocyte dysmorphisms for ICSI patients may prove relevant to the outcome in subsequent patient cycles. Human Reproduction 2001;16:21182123.

10 Esfandiari N, Ryan EA, Gotlieb L, Casper RF: Successful pregnancy following transfer of embryos from oocytes with abnormal zona pellucida and cytoplasm morphology. Reprod Biomed Online 2005;11:620623.

11 Balaban B, Urman B, Sertac A, Alatas C, Aksoy S, Mercan R: Oocyte morphology does not affect fertilization rate, embryo quality and implantation rate after intracytoplasmic sperm injection. Hum Reprod 1998;13:3431-3433.

12 De Sutter P, Dozortsev D, Qian C, Dhont M: Oocyte morphology does not correlate with fertilization rate and embryo quality after intracytoplasmic sperm injection. Hum Reprod 1996;11:595-597. 


\section{Cellular Physiology Cell Physiol Biochem 2016;39:677-684

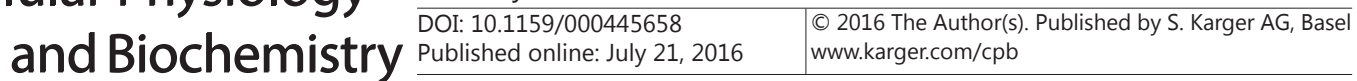 \\ Xu et al.: High FSH Caused Brown Oocyte and a Lower Pregnacy Rate}

13 Balaban B, Ata B, Isiklar A, Yakin K, Urman B: Severe cytoplasmic abnormalities of the oocyte decrease cryosurvival and subsequent embryonic development of cryopreserved embryos. Hum Reprod 2008;23:1778-1785.

14 Esfandiari N, Burjaq H, Gotlieb L, Casper RF: Brown oocytes: implications for assisted reproductive technology. Fertil Steril 2006;86:1522-1525.

15 Shi W, Xu B, Wu LM, Jin RT, Luan HB, Luo LH, Zhu Q, Johansson L, Liu YS, Tong XH: Oocytes with a dark zona pellucida demonstrate lower fertilization, implantation and clinical pregnancy rates in IVF/ICSI cycles. PLoS One 2014;9:e89409.

16 Wu YT, Wang TT, Chen XJ, Zhu XM, Dong MY, Sheng JZ, Xu CM, Huang HF: Bone morphogenetic protein-15 in follicle fluid combined with age may differentiate between successful and unsuccessful poor ovarian responders. Reprod Biol Endocrinol 2012;10:116.

17 Ziebe S, Petersen K, Lindenberg S, Andersen AG, Gabrielsen A, Andersen AN: Embryo morphology or cleavage stage: how to select the best embryos for transfer after in-vitro fertilization. Hum Reprod 1997;12:1545-1549.

18 Sanchez F, Adriaenssens T, Romero S, Smitz J: Different follicle-stimulating hormone exposure regimens during antral follicle growth alter gene expression in the cumulus-oocyte complex in mice. Biol Reprod 2010;83:514-524.

19 O'Callaghan D, Yaakub H, Hyttel P, Spicer LJ, Boland MP: Effect of nutrition and superovulation on oocyte morphology, follicular fluid composition and systemic hormone concentrations in ewes. J Reprod Fertil 2000;118:303-313.

20 Li M, Zhao Y, Zhao CH, Yan J, Yan YL, Rong L, Liu P, Feng HL, Yu Y, Qiao J: High FSH decreases the developmental potential of mouse oocytes and resulting fertilized embryos, but does not influence offspring physiology and behavior in vitro or in vivo. Hum Reprod 2013;28:1309-1323.

21 Rankin T, Talbot P, Lee E, Dean J: Abnormal zonae pellucidae in mice lacking ZP1 result in early embryonic loss. Development 1999;126:3847-3855.

22 Monne M, Han L, Jovine L: Tracking down the ZP domain: From the mammalian zona pellucida to the molluscan vitelline envelope. Semin Reprod Med 2006;24:204-216.

23 Peluso C, Fonseca FL, Gastaldo GG, Christofolini DM, Cordts EB, Barbosa CP, Bianco B: AMH and AMHR2 polymorphisms and AMH serum level can predict assisted reproduction outcomes: a cross-sectional study. Cell Physiol Biochem 2015;35:1401-1412.

24 Zhao C, Liu X, Shi Z, Zhang J, Jia X, Ling X: Role of serum miRNAs in the prediction of ovarian hyperstimulation syndrome in polycystic ovarian syndrome patients. Cell Physiol Biochem 2015;35:10861094.

25 Trevisan CM, Peluso C, Cordts EB, de Oliveira R, Christofolini DM, Barbosa CP, Bianco B: Ala307Thr and Asn680Ser polymorphisms of FSHR gene in human reproduction outcomes. Cell Physiol Biochem 2014;34:1527-1535.

26 Palmstierna M, Murkes D, Csemiczky G, Andersson O, Wramsby H: Zona pellucida thickness variation and occurrence of visible mononucleated blastomers in preembryos are associated with a high pregnancy rate in IVF treatment. J Assist Reprod Genet 1998;15:70-75.

27 Zhou HX, Ma YZ, Liu YL, Chen Y, Zhou CJ, Wu SN, Shen JP, Liang CG: Assessment of mouse germinal vesicle stage oocyte quality by evaluating the cumulus layer, zona pellucida, and perivitelline space. PLoS One 2014;9:e105812. 